\title{
A Neural-Genetic Technique for Coastal Engineering: Determining Wave-induced Seabed Liquefaction Depth
}

\author{
Daeho Cha ${ }^{1}$, Michael Blumenstein ${ }^{1}$, Hong Zhang ${ }^{1}$ and Dong-Sheng Jeng ${ }^{2}$ \\ ${ }^{1}$ Griffith University Gold Coast Campus, PMB 50 Gold Coast Mail Centre QLD \\ 9726, Australia \\ f.cha@griffith.edu.au, \\ M.Blumenstein@griffith.edu.au, Hong.Zhang@griffith.edu.au, \\ ${ }^{2}$ University of Sydeney NSW 2006, Australia \\ d.jeng@civil.usyd.edu.au
}

\begin{abstract}
In the past decade, computational intelligence (CI) technologies have been widely adopted in various fields such as business, science and engineering, as well as information technology. Specifically, hybrid techniques using artificial neural networks (ANNs) and genetic algorithms (GAs) are becoming an important alternative for solving problems in the field of engineering in comparison to traditional solutions, which ordinarily use complicated mathematical theories. The wave-induced seabed liquefaction problem is one of most critical issues for analysing and designing marine structures such as caissons, oil platforms and harbours. In the past, various investigations into wave-induced seabed liquefaction have been carried out including numerical models, analytical solutions and some laboratory experiments. However, most previous studies are based on complicated mathematical theories. In this study, the proposed a neural-genetic model will be applied to wave-induced liquefaction, provide a better prediction of liquefaction potential. The ANN+GA simulation results illustrate the applicability of a hybrid, neural-genetic technique for the accurate prediction of wave-induced liquefaction depth, which can also provide coastal engineers with alternative tools to analyse the stability of marine sediments.
\end{abstract}

\section{Introduction}

\subsection{Artificial Neural Networks in Engineering}

Artificial Neural Networks (ANNs) are amongst the most successful empirical processing technologies to be used in engineering applications. ANNs serve an 
important function for engineering purposes such as modelling and predicting the evolution of dynamic systems.

Hagan et al. [9] espoused that the pioneering work in neural networks commenced in 1943 when McCulloch and Pitts [10] postulated a simple mathematical model to explain how biological neurons work. It may be the first significant publication on the theory of artificial neural networks, which is generally considered. ANN models have been widely applied to various engineering problems. For example, the prediction of water quality parameters [1], generation of wave equations based on hydraulic data [2], soil dynamic amplification analysis [3], tide forecasting using artificial neural networks [4], prediction of settlement of shallow foundations [5], earthquake-induced liquefaction [6], and ground settlement by deep excavation [7].

Unlike conventional approaches based on engineering mechanics, the main requirement for accurate prediction using ANN models is an appropriate database. With sufficient training data, ANNs can provide accurate predictions for various engineering problems.

\subsection{Genetic Algorithms}

Generally speaking Genetic Algorithms (GAs) are one of the various Computatonal Indtelligence (CI) technologies, which also include ANNs and Fuzzy Logic.

Fundamental theories of GAs were established by John Holland in the early 1970s [8]. John Holland [8] was amongst the first to put computational evolution on a firm theoretical footing. The GA's main role is numerical optimisation inspired by natural evolution. GAs can be applied to an extremely wide range of problems. The basic component of GAs is strings of binary values (sometimes real-values) called chromosomes. A GA operates on a population of individuals (chromosomes), each presenting a possible solution to a given problem. Each chromosome is assigned a fitness value based on a fitness function, and its operation is based on crossover, selection and mutationd.

\subsection{ANN models trained by GAs (Evolutionary Algorithms)}

Generally, it is time-consuming to configure and adjust the settings of ANN models during the supervised training procedure e.g. those using the Backpropagation (BP) algorithm. Even though its results may be acceptable for some engineering applications, ANN training algorithms such as BP may suffer problems inherent in gradient descent-based techniques such as being trapped in local minima and an incapability of finding a global minimum if the error function is multi-modal and/or non-differentiable [11]. An ANN model trained using GAs can deal with large, complicated spaces, which are on occasion nondifferentiable, and multi-modal as is common in real world problems. Hence, the use of a hybrid technique combining GAs in conjunction with ANNs is 


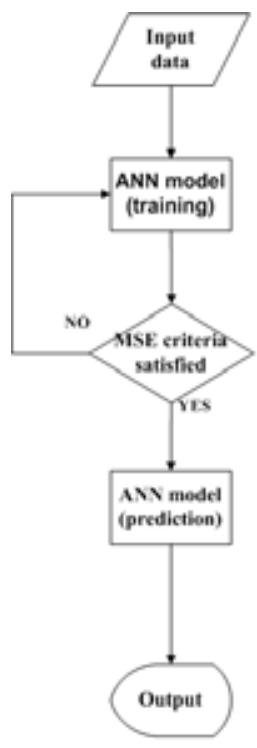

(a)

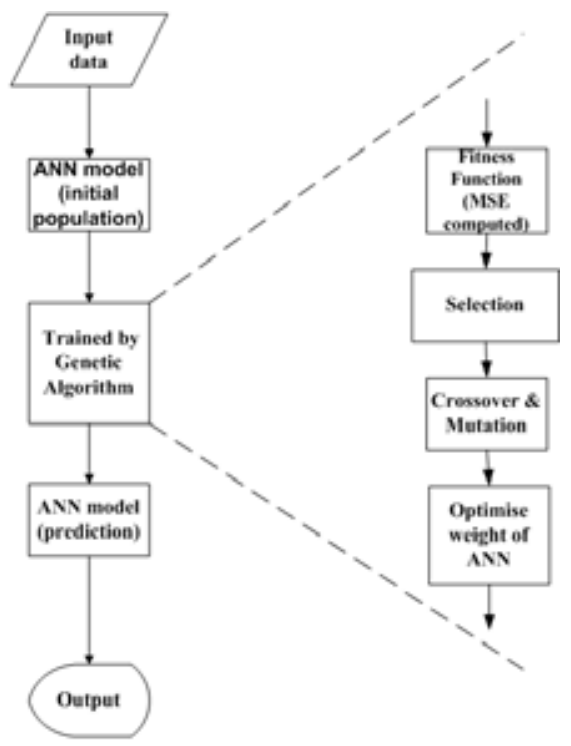

(b)

Fig. 1. Comparison of a traditional ANN model (a) and an ANN model trained by GAs (b).(MSE: Mean Square Error)

Give a unique label

advantageous in complex engineering problems. The configuration of an ANN model using GAs is shown in Figure 1(b).

ANN models predict data, based on the relationship between input and output values. This relationship is based on the iterative modification of network weights, from the training procedure. Clearly, the training procedure is key factor of an ANN model. However, aside from the inherent problems associated with gradient descent-based techniques (as described above) the training procedure can be time consuming in terms of selecting the most appropriate settings for ANN training; therefore it is beneficial to employ GAs to optimise an ANN model's weights in a hybrid technique for an application such as wave-induced seabed liquefaction.

\subsection{Wave-induced seabed liquefaction}

In the last few decades, various investigations of wave-induced seabed liquefaction have been carried out. Although the protection of marine structures has been extensively studied in recent years, understanding of their interaction with waves and the seabed is far from complete. Damage of marine structures still occurs from time to time, with two general failure modes evident. The first mode is that of structural failure, caused by wave forces acting on and damaging the structure itself. The second mode is that of foundation failure, 
caused by liquefaction or erosion of the seabed in the vicinity of the structure, resulting in collapse of the structure as a whole. The numerous research studies carried on this topic last decade. [12][13]. Figure2 illustrates the change of a seabed due to wave action (Fig 2(a): without liquefaction, Fig 2(b) with liquefaction).

Bjerrum [14] was possibly the first author that considered wave-induced liquefaction occurring in saturated seabed sediments. Later, Nataraja et al. [15] suggested a simplified procedure for ocean-based, wave-induced liquefaction analysis. Recently, Rahman [16] established the relationship between liquefaction and characteristics of wave and soil. He concluded that liquefaction potential increases in degree of saturation and with an increase of wave period. Jeng [17] examined wave-induced liquefied state for several different cases, together with Zen and Yamazaki's [18] field data. He found that no liquefaction occurs in a saturated seabed, except in very shallow water, for large waves and a seabed with very low permeability. For more advanced poro-elastic models for the wave-induced liquefaction potential, the readers can refer to Sassa and Sekiguchi [19] and Sassa et al. [20]. All aforementioned investigations have been reviewed by Jeng [21]. However, most previous investigations for the wave-induced liquefaction potential in a porous seabed have been based on various assumptions of engineering mechanics, which limits the application of the model in realistic engineering problems.

The major difference between traditional engineering mechanics approaches and CI models (ANNs and GAs) for the estimation of the wave-induced liquefaction is the procedure. Conventional models for wave-induced liquefaction procedures always involve complicated mathematical calculations with numerous variables, such as shear modulus, degree of saturation and Poisson ratio etc. However, CI models which based on statistical theory, can be built and learn the knowledge of a database with less complicated governing equations compare with tradtional engineering approach.

In this study, we adopt a neural-genetic model for the wave-induced seabed maximum liquefaction, based on a pre-built poro-elastic model [22].

\section{A neural-genetic technique for wave-induced liquefaction}

Besides the use of standard ANNs for wave-induced liquefaction depth prediction, we will discuss a neural-genetic approach for this problem. Generally, there are two areas where GAs have been used in ANN modelling, these are: optimising the weights of network connections (training) [30] and optimising neural network structure design [23][24][11]. In this study we adopted the option of optimising network weights using a single hidden layer feedforward ANNs (Figure 3).

As shown in Figure 3, a multi-layer network is an expansion of the single layer network, and it can be used to solve more difficult and complicated prob- 


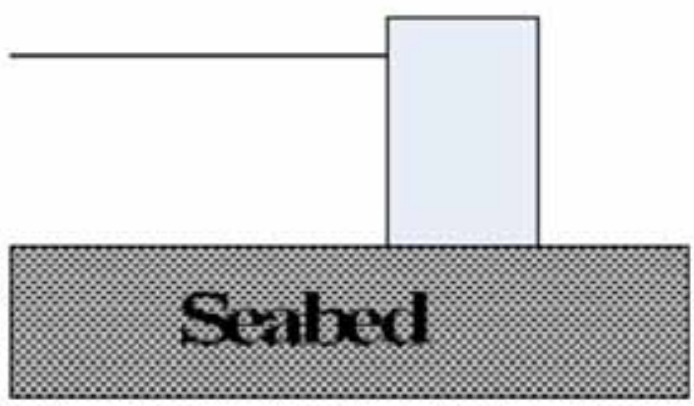

(a) Seabed without liquefaction

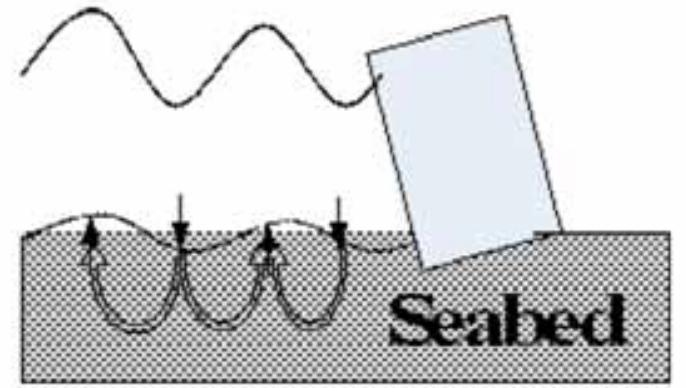

(b) Seabed with liquefaction

Fig. 2. Phenomenon of wave-induced seabed liquefaction 


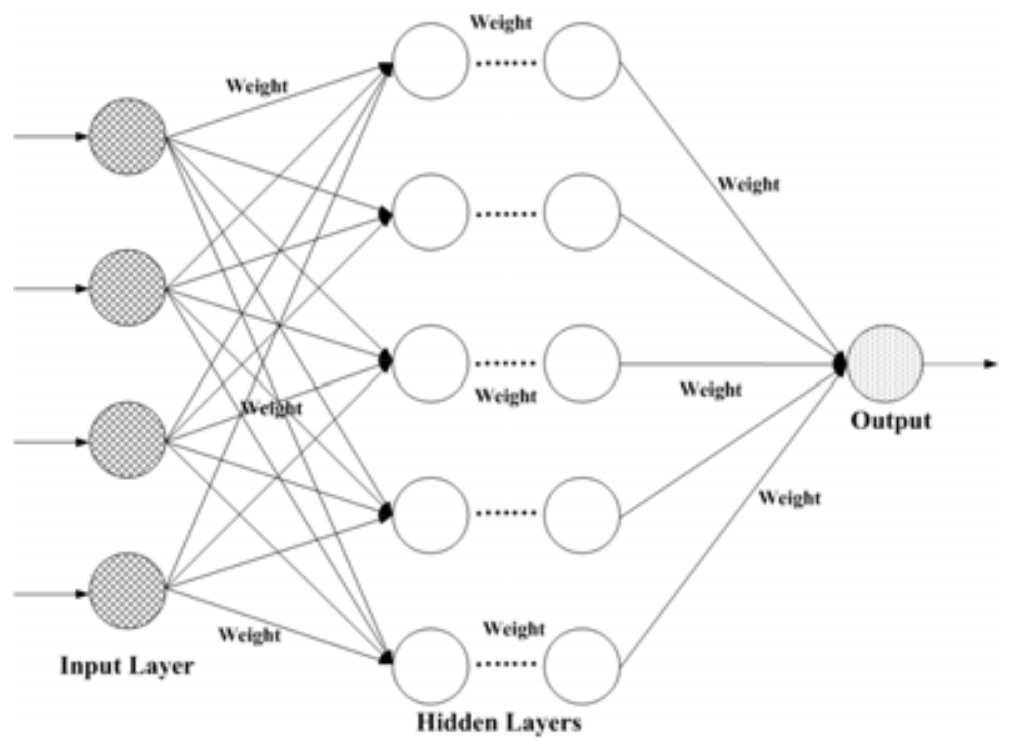

Fig. 3. A typical multi-layer feedforward network architecture.

lems. It consists of an input layer, one or more hidden layers of neurons and an output layer of neurons. In the present study, GAs are utilised to calculate the weights of the network and adjust the interconnections to minimise its output error. It can be applied to the network, which has at least one hidden layer, and fully connected to all units in each layer. The goal of this procedure is to obtain a desired output when certain inputs are given. The general network error function is shown in (1), where $D_{x}$ and $O_{x}$ are respectively the desired and actual output value.

$$
E(x)=\frac{1}{n} \sum_{x=1}^{n}\left(D_{x}-O_{x}\right)^{2}
$$

Since the error is the difference between the actual output and the target output, the error depends on the weights, so we employed this error function as the GA fitness function for optimising the weights instead of standard, iterative, gradient descent-based training (2).

$$
f(x)=E_{\max }-E(x)
$$

where, $f(x)$ is the corresponding fitness and $\% E_{\max }$ is maximum performance error.

For the GA selection function, we used the Roulette wheel method developed by Holland [8]. It is defined as 


$$
P_{i}=\frac{F_{i}}{\sum_{i=1}^{S} F_{i}}
$$

where, $P_{i}$ is the probability for each individual chromosome, $S$ is the population size, and $F_{i}$ is the fitness value of each chromosome.

In the use of GAs, there are basic operators employed / modified in the search mechanism, which are Crossover and Mutation. For example, the basic two point Crossover operator takes two individuals from a chromosome and produces two new individuals, whilst mutation alters one individual to produce a single new solution. Further discussion and details pertaining to Crossover and Mutation settings in this research are presented in the results section.

\subsection{Data preparation}

To ensure the accurate prediction of an ANN model trained using GAs, we needed to build a reliable database or training/test sets. In this section, we describe the establishment of the database by an existing poro-elastic model developed by the author [25].

\section{Poro-elastic model}

In this model, we consider an ocean wave propagating over a porous seabed of infinite thickness. A two-dimensional wave-seabed interaction problem is considered, treating the porous seabed as hydraulically isotropic with a uniform permeability. Biot [26] presented a general set of equations governing the behaviour of a linear elastic porous solid under dynamic conditions. They are summarized in the tensor form as below

$$
\begin{gathered}
\sigma_{i j, j}=\rho \ddot{u}_{i}+\rho_{f} \ddot{w}_{i} \\
-p,,_{i}=\rho_{f} \ddot{u}+\frac{\rho_{f}}{n} \ddot{w}_{i}+\frac{\rho_{f} g}{k_{z}} \dot{w}_{i} \\
\dot{\varepsilon}_{i i}+\dot{w}_{i i}=-\frac{n}{K_{f}} \dot{p}
\end{gathered}
$$

where $p$ is pore pressure, $n$ is porosity, $\rho$ is the combined density, $\rho_{f}$ is the fluid density, $u$ and $w$ are the displacements of solid and relative displacements of solid and pore fluid. $\frac{1}{K_{f}}$ is the compressibility of pore-fluid, which is defined by

$$
\frac{1}{K_{f}}=\frac{1}{2 \times 10^{9}}+\frac{1-S}{P_{w o}}
$$

where $S$ is the degree of saturation, $P_{w o}$ is the absolute water pressure.

The definition of effective stresses, $\sigma_{i j}^{\prime}$, which are assumed to control the deformation of the soil skeleton, given by the total stress $\left(\sigma_{i j}\right)$ and pore pressure $(p)$ as, 


$$
\sigma_{i j}=\sigma_{i j}^{\prime}-\delta_{i j} p
$$

where, $\delta_{i j}$ is the Delta denotation.

Therefore, the equation of force balance, equation (4) becomes

$$
-\left(\frac{n}{K_{f}} \dot{p}\right)_{i}=\left(\dot{\varepsilon}_{i i}+\dot{w}_{i i}\right)_{i} \Rightarrow-p,{ }_{i}=\frac{K_{f}}{n}\left(\varepsilon_{i i}+w_{i i}\right)_{i} .
$$

Then substituting (9) into (4) and (6), the governing equation can be rewritten as

$$
\frac{K_{f}}{n}\left(\varepsilon_{i i}+w_{i i}\right)_{i}=\rho_{f} \ddot{u}_{i}+\frac{\rho_{f}}{n} \ddot{w}_{i}+\frac{\rho_{f} g}{k_{z}} \dot{w}_{i} .
$$

If the acceleration terms are neglected in the above equation, it becomes the consolidation equation, which has been used in previous work [17]. Based on the wave-induced soil and fluid displacements, we can obtain the waveinduced pore pressure, effective stresses and shear stresses. Detailed information of the above solution can be found in [25].

\section{Estimation of liquefaction}

It has generally been accepted that when the vertical effective stress vanishes, the soil will be liquefied. Thus, the soil matrix loses its strength to carry and load and consequently causes seabed instability. Based on the concept of excess pore pressure, Zen and Yamazaki [18] proposed a criterion of liquefaction, which has been further extended by considering the effects of lateral loading $[17]$

$$
-\frac{1}{3}\left(1+K_{0}\right)\left(\gamma_{s}-\gamma_{w}\right) z+\left(P_{b}-p\right) \leq 0 .
$$

where $K_{0}$ is the coefficient of earth pressure at rest, which is normally varid from 0.4 to 1.0 , and 0.5 is commonly used for marine sediments [28], $\gamma_{s}$ is teh unit weight of soil, $\gamma_{w}$ is the unit weight of water, $p$ is porepressure, $z$ is maximum liquefaction depth and $P_{b}$ is the wave pressure at the seabed surface, which is given by

$$
p=\frac{\gamma_{w} H}{\cosh k d} \cos (k x-\omega t)
$$

where " $\cos (k x-\omega t)$ " denotes the spatial temporal variations in wave pressure within the two-dimensional progressive wave described above. $p_{o}$ is the amplitude of the wave pressure on the Stokes wave theory to a first-order. 


\section{Results and discussion}

\subsection{Neural-genetic model configuration for wave-induced liquefaction}

In general, wave-induced seabed liquefaction is often calculated by complicated mathematical procedures such as poro-elastic models. However, an ANN-based model does not need to solve complicated nonlinear equations; rather it requires high quality input data and accurate values for output data. As pointed out in previous work [17], the most important factors, which significantly affect the wave-induced soil liquefaction, include the degree of saturation, seabed thickness, soil permeability, wave period, water depth and wave height. Since permeability has been very sensitive to the occurrence of the wave-induced liquefaction potential, we use a fixed value of permeability in addition to the other parameters as inputs to the ANN model. The waveinduced maximum liquefaction depth is the output value. Figure 4 illustrates the structure of the ANN model for this study. The important factors of this ANN model are tabulated in Table 1.

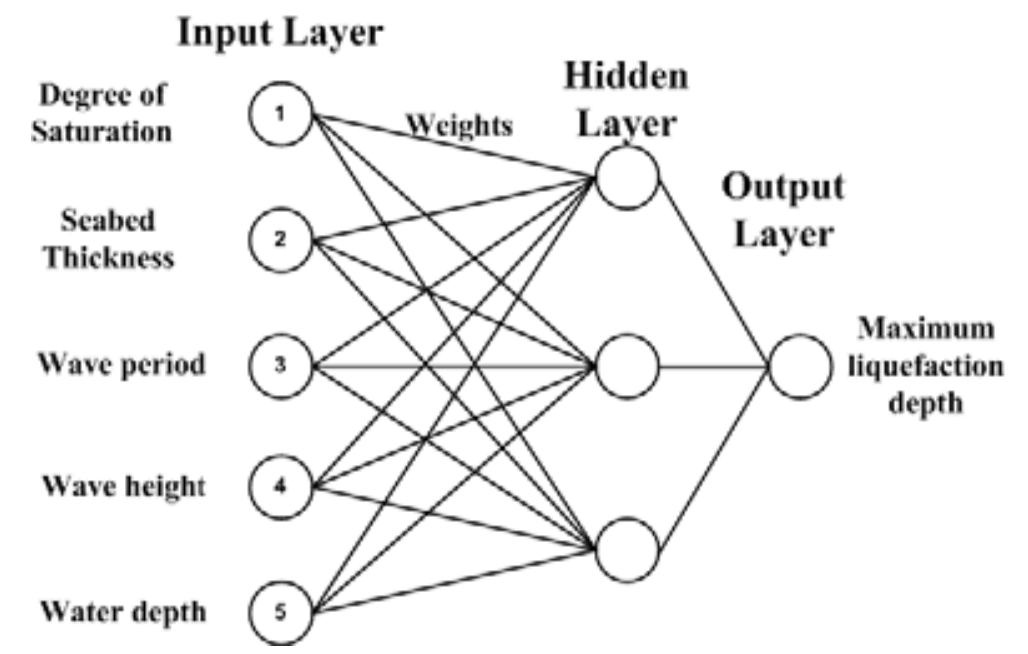

Fig. 4. Structure of ANN model for wave-induced liquefaction.

The database generated by the Poro-elastic model is used to establish the ANN model, as shown in Table 2. As seen in the table, we built the database, based on most possible ranges of wave and soil conditions. Among this input data, we choose most affective componet of wave-induced liquefaction, which included soil permeability, seabed thickness, degree of saturation, wave period, wave height and water depth for the ANN+GA model. 
Table 1. key components of neural network model

\begin{tabular}{ll}
\multicolumn{2}{c}{ Training model } \\
\hline Number of input neurons & 5 \\
Number of output neurons & 1 \\
Number of hidden neuron & 5 or various \\
learning rate & 0.5 \\
momentum factor & 0.2 \\
\hline
\end{tabular}

Table 2. Input data for the poro-elastic model

\begin{tabular}{ll}
\multicolumn{2}{c}{ Wave characteristics } \\
\hline wave period $(T)$ & $8 \mathrm{sec}$, various \\
wave height $(H)$ & $7.5 \mathrm{~m}$, various \\
water depth $(d)$ & $50 \mathrm{~m}$, various \\
\hline \multicolumn{2}{c}{ Soil characteristics } \\
\hline soil permeability $\left(K_{z}\right)$ & $10^{-4}, 5 \times 10^{-4}$ \\
seabed thickness $(h)$ & $10 \mathrm{~m}$, various \\
shear modulus $(G)$ & $10^{7} \mathrm{~N} / \mathrm{m}^{2}$ \\
Poisson's ratio $(\mu)$ & 0.4 \\
Porosity $(n)$ & 0.4 \\
degree of saturation $(S)$ & 0.95 various \\
\hline
\end{tabular}

In the current study, we had approximately 20,000 outputs of maximum liquefaction depth from the each numerical model. Among these data we used 80training procedure, and the remaining data for validating the prediction capability.

In this paper, we not only use a correlation value $\left(R^{2}\right)$ for comparison of the analytical Poro-elastic model and the neural-genetic model but we also use the Root Mean Square Error (RMSE) value. The RMSE is defined as

$$
R M S E=\sqrt{\frac{1}{N} \sum_{i=1}^{N}\left(L_{A i}-L_{P i}\right)^{2}}
$$

where $L_{A i}$ and $L_{P i}$ are the liquefaction depth from the ANN model and the Poro-elastic model respectively; $\mathrm{N}$ is the total number of liquefaction depth data.

\subsection{ANN model training using GAs for wave-induced liquefaction}

Generally, it is time-consuming to configure and adjust the settings of an ANN model during the supervised training procedure. Even though its results are acceptable from the engineering viewpoint, an ANN model trained using GAs can reduce the complexity of the procedure; hence, it is an advantage to use them in conjunction with ANNs. 
As discussed in the previous sections, ANN models predict data, based on the relationship between input and output values. This relationship is based on the iterative modification of network weights, from the training procedure. It clearly shows that the training procedure is a key factor of an ANN model. However, the authors found that the training procedure can be time consuming in terms of selecting the most appropriate settings for ANN training; therefore the authors propose to use GAs to optimise the ANN model's weights, which is described in the previous section. The use of GAs is advantageous in this research due to the problems inherent in regular gradient-descent based learning techniques (as discussed in Section 1.4).

Hence to initiate the training procedure, we save the first weight configuration of the existing ANN model (small random values) for the initial GA population.

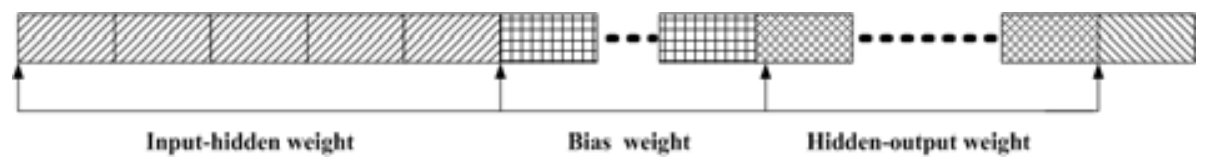

Fig. 5. The concept of the chromosome used in the neural-genetic approach.

Figure 5 illustrates the concept of a chromosome, which we adopted (encoded) from the ANN model.

As seen in Figure 5, the size of the chromosome depends on the number of weights in the ANN model. After we save the initial population from the ANN model, GA operations are performed, such as selection, crossover and mutation for optimising the weights.

In this study, we adopted 3 crossover (simple, arithmetic and heuristic crossover) and mutation (uniform, non-uniform and boundary mutation) functions, which were developed by [29]. For example, let ai and bi be the lower and upper bound, respectively, for each variable i then Uniform mutation is described as below

$$
x_{i}^{\prime}= \begin{cases}U\left(a_{i}, b_{i}\right), & \text { if } \mathrm{i}=\mathrm{j} ; \\ x_{i}, & \text { otherwise }\end{cases}
$$

where, $U\left(a_{i}, b_{i}\right)$ is uniform random number, $j$ is a randomly selected variable Similarly Non-uniform mutation is described as

$$
x_{i}^{\prime}= \begin{cases}x_{i}+\left(b_{i}-x_{i}\right) f(G), & \text { if } \mathrm{r}_{i} \leq 0.5 \\ x_{i}-\left(x_{i}+a_{i}\right) f(G), & \text { if } \mathrm{r}_{i} \geq 0.5, \\ x_{i}, & \text { otherwise }\end{cases}
$$

where,$f(G)=\left(r_{2}\left(1-\frac{G}{G_{\max }}\right)\right)^{b} r_{1}$ and $r_{2}$ is a uniform random number between $(0,1), G$ is the current generation, $G_{\max }$ is the maximum number of generations, $b$ is a shape parameter. 


\subsection{Results for determining wave-induced liquefaction}

Numerous experiments were conducted to determine the maximum liquefaction depth for two conditions of soil permeability. In this sub-section, the top results are presented in Figures 3.3 and 3.3 using the neural-genetic approach with accompanying discussion.

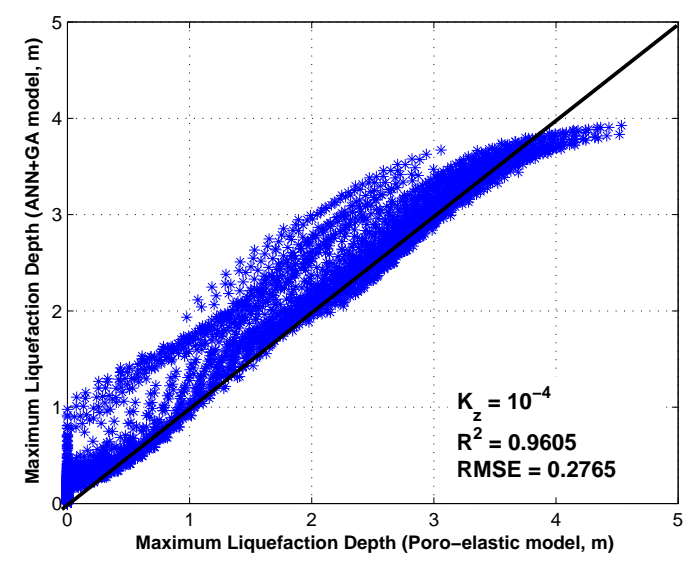

(a) 6000 Generations

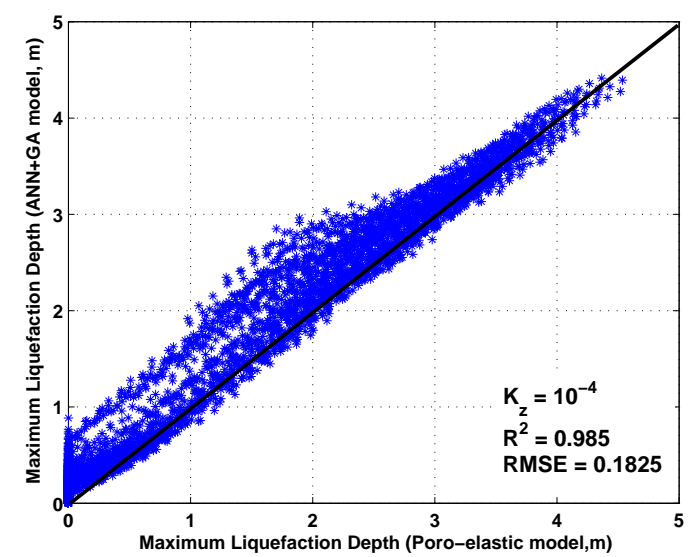

(a) 8000 Generations

Figure 3.3 represents the predicted maximum liquefaction depth obtained from an ANN model trained by GAs versus the poro-elastic numerical maximum liquefaction depth (soil permeability, $10^{-4} \mathrm{~m} / \mathrm{s}$ ). As seen in the figures, 


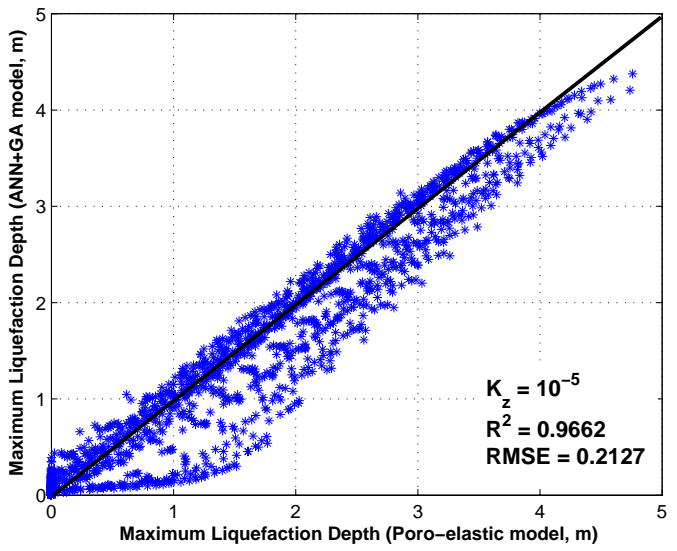

(a) 6000 Generations

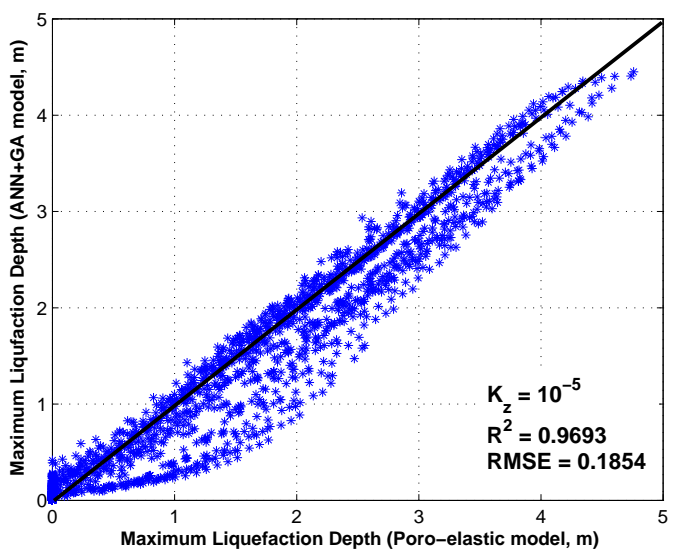

(a) 8000 Generations

overall, the prediction of maximum liquefaction depth agrees with the numerical calculation data. It is shown that the correlation of the ANN model and the poro-elastic model is over $96 \%$. The figure illustrates that the RMSE value is between the 10 to $30 \%$ range, which is acceptable for an engineering application. Also, the figures indicated that the correlation of the neural-genetic model with the poro-elastic model and RMSE values could improve depending on the GA settings used (e.g. Fig 3.3 (a) 6000 total generations, Fig 3.3 (b) 8000 total generations).

Figure 3.3 illustrates the predicted maximum liquefaction depth obtained using the neural-genetic model versus the poro-elastic numerical maximum liquefaction depth (soil permeability, $5 \times 10^{-4} \mathrm{~m} / \mathrm{s}$ ). As shown in the above 
Table 3. GA setting employed for training

figures, prediction of maximum liquefaction depth using the neural-genetic model agrees well the with the poro-elastic model, in that the correlation values are greater than 96 less than $25 \%$ in both cases. Figure 7 results are slightly better than those shown in Figure 6 because the GA operation settings were based on those in Fig 3.3(b). It is clearly shown that better results may be produced if we vary the GA settings, with specific attention to increasing the number of generations and varying crossover and mutation parameters. These results indicated that the performance of $\mathrm{ANN}+\mathrm{GA}$ model for the prediction of maximum wave-induced seabed liquefaction can comparable with previous authors results [27]. In this study we adopted 3 crossover and mutation funtion which all used in the ANN+GA model. The details of these settings are displayed in Table 3 .

\section{Conclusions}

In this study, we adopted the concept of GA-based training of ANN models in an effort to overcome the problems inherent in some ANN training procedures (i.e. gradient-based techniques) whilst providing accurate results for determining maximum liquefaction depth in a real-world application.

Unlike the conventional engineering mechanics approaches, the neuralgenetic technique does not require a complicated mathematical procedure, and saves time for configuring and adjusting the settings of an ANN model during the supervised training process.

In the proposed neural-genetic model, several important parameters, including wave period, water depth, wave height, seabed thickness and the degree of saturation, were used as the input parameters with constant soil permeability, whilst the maximum liquefaction depth was the output parameter. Experimental results demonstrate that the neural-genetic model is successful in predicting the wave-induced maximum liquefaction depth.

\section{References}

1. Maier H R and Dandy H R (1997) Modeling cyanobacteria (blue-green algae) in the River Murray using artificial neural networks, Mathematics and Computers in Simulation 43:377-386.

2. Dibike Y B, Minns A W and Abbott M B (1999) Applications of artificial neural networks to the generation of wave equations from hydraulic data, Journal of Hydraulic Research 37(1):81-97. 
3. Hurtado J E, Londono J E and Meza J E (2001) On the applicability of neural networks for soil dynamic amplification analysis, Soil Dynamics and Earthquake Engineering 21(7):579-591.

4. Lee T L and Jeng D S (2002) Application of artificial neural networks in tide forecasting. Ocean Engineering 29(9):1003-1022.

5. Mohamed A S, Holger R M and Mark B J (2002) Predicting Settlement of Shallow Foundations using Neural Networks. Journal of Geotechnical and Geo environmental Engineering 128(9):785-793.

6. Jeng D S, Lee T L and Lin C (2003) Application of artificial neural networks in assessment of Chi-Chi earthquake-induced liquefaction. Asian Journal of Information Technology 2(3):190-198.

7. Leo S S and Lo H S (2004) Neural Network based regression model of ground surface settlement induced by deep excavation, Automation in Construction 13:279-289.

8. Holland J (1975) Adaptation in Natural and Artificial Systems, University of Michigan Press. (Second edition: MIT Press, 1999)

9. Hagan M T, Demuth H B, and Beale M (1996) Neural Network Design, PWS Publishing, Boston, MA.

10. McCulloch W S, Pitts W (1943) A logical calculus of the ideas imminent in nervous activity, Bulletin and Mathematical Biophysics 5:115-133.

11. Yao X (1999) Evolving Artificial Neural Networks, Proceedings of the IEEE 87(9):1423-1447.

12. Zen K, Umehara Y, Finn W D L (1985) A case study of the wave-induced liquefaction of sand layers under damaged breakwater, In: Proceedings 3rd Canadian Conference on Marine Geotechnical Engineering 505-520.

13. Silvester R, Hsu J R C (1989) Sines Revisited. Journal of Waterways, Port, Coastal and Ocean Engineering, A.S.C.E 1153:327-344.

14. Bjerrum J (1973) Geotechnical problem involved in foundations of structures in the North Sea. Geotechnique 233:319-358.

15. Nataraja M S, Singh H, Maloney D (1980) Ocean wave-induced liquefaction analysis: a simplified procedure. In: Proceedings of an International Symposium on Soils under Cyclic and Transient Loadings 509-516.

16. Rahman M S (1997) Instability and movement of ocean floor sediments. A review. International Journal of Offshore and Polar Engineering 73:220-225.

17. Jeng D S (1997) Wave-induced seabed instability in front of a breakwater. Ocean Engineering 24(10):887-917.

18. Zen K, Yamazaki H (1991) Field observation and analysis of wave-induced liquefaction in seabed. Soils and Foundations 31(4):161-179.

19. Sassa S and Sekiguchi H (2001) Analysis of wave-induced liquefaction of sand beds. Geotechnique 51(2):115-126.

20. Sassa S, Sekiguchi H and Miyamamoto J (2001) Analysis of progressive liquefaction as moving-boundary problem. Geotechnique 51(10):847-857.

21. Jeng D S (2003) Wave-induced seafloor dynamics. Applied Mechanics Review 56(4):407-429.

22. Jeng D S and Cha D H(2003) Effects of dynamic soil behaviour and wave nonlinearity on the wave-induced pore pressure and effective stresses in porous seabed. Ocean Engineering 30(16):2065-2089.

23. Montana D J and Davis L (1989) Training Feedforward neural networks using genetic algorithms, Proceedings of the International Joint Conference on Artificial Intelligence 762-767. 
24. Miller G F, Todd P M and Hegde P M (1989) Designing neural networks using genetic algorithms, Proceedings of the Third International Conference on Genetic Algorithms. San Francisco: Morgan Kaufmann Publishers.

25. Cha D H (2003) Mechanism of Ocean Waves Propagating over a Porous Seabed. MPhil Thesis, Griffith University, Australia.

26. Biot M A (1956) Theory of propagation of elastic waves in a fluid-saturated porous solid. Part I: Low frequency range; Part II. High frequency analysis. Journal of Acoustics Society 28:168-191.

27. Jeng D S, Cha D H and Michael B (2004) Neural network model for the prediction of wave-induced liquefaction potential Ocean Engineering 31(17-18):20732086.

28. Scott, R F (1968) Principle of Soil Mechanics, Addison-Publishing, Massachussetts.

29. Michalewicz Z (1994) Genetic Algorithms + Data Structures = Evolution Programs, AI Series, Springer-Verlag, New York.

30. Rooij van A J F, Jain L C and Johnson R P (1996) Neural Network Training using Genetic Algorithms, World Scientific Publishing, London. 